\title{
STRUCTURE OF THE ATOMIC NUCLEUS
}

A

BOUT three hundred nuclear physicists, a hundred and twenty of them from overseas, met at the University of Birmingham during July 13-18 for discussions on nuclear structure. At each session, an opening speaker surveyed some portion of the field: his introduction was followed by contributions from those who had new information or who took other points of view. Much of the value of such a conference lies in the debating of divergent opinions or apparently contradictory experimental facts, but a report after the style of Hansard is not possible. The following account, covering only a few of the topics actually discussed, is intended to pick out some of the points of liveliest interest.

In several of the sessions the question of nuclear models was raised. In particular, this referred to the suitability of the 'shell model', as opposed to the 'liquid drop' model. The first treats the nucleus, as is usual in the case of the atom, as an assembly of individual particles, moving independently in a common field of force. For the past few years considerable success has been obtained from the use of this model, provided one assumes that there exists for each particle a strong interaction between its spin and its orbital angular momentum. B. H. Flowers (Harwell) summed up this picture in one of the opening talks and showed that, by making use of the correct alignment of the orbits of different particles when there is more than one particle outside a closed shell, one can account for the behaviour of magnetic moments and quadrupole moments in cases which had previously presented difficulty. Many other successes of the shell model, relating also to nuclear reactions, were referred to in the discussion.

On the other hand, it was stressed by A. Bohr (Copenhagen) that an approach starting from a collective motion of many particles also finds a good deal of support from direct evidence. He assumes that the potential field acting on all the particles has a fairly sharply defined surface which may alter its shape as a result of the motion of the particles, and which therefore represents a set of degrees of freedom of the system. Since this surface is coupled with the motion of each particle inside it, it provides an indirect means of connecting the motion of different particles.

One particular piece of evidence favouring this 'liquid drop' model is the existence of 'rotational' states in certain nuclei. To take the simplest case, an even-even nucleus with angular momentum zero in its ground-state, often is found to have excited states of angular momenta 2, 4 ..., the energy of excitation being proportional to $\dot{J}(\dot{J}+1)$, where $J$ is the angular momentum. The factor of proportionality defines an effective moment of inertia, and this is found to be much larger than that for a single nucleon in an orbit the size of a nucleus, but much smaller than that for the whole nucleus considered as a rigid body. According to the Bohr theory, these states are to be understood not as a rotation of the nucleus as a whole but as an irrotational mode of motion in which the shape of the external surface revolves about its centre. As this model predicts, the transitions between such levels are associated with very large electric quadrupole moments which would not easily be explained in other ways. T. Huus (Copenhagen) described recently published experiments in which excited states, interpreted as rotational levels, are produced by the electric field of protons passing close to nuclei but not actually colliding.

In one sense the liquid drop model is a different extreme to the shell model, and the use of these different pictures in itself does not necessarily constitute a contradiction. In particular, the liquid drop model does not allow for the peculiar features arising from the closing of shells and should be most satisfactory well away from the closed-shell nuclei (magic numbers), whereas the shell model becomes prohibitively complicated in that region and allows the most precise predictions near the closed shells.

However, differences of opinion arise when one has to decide which of the two pictures is appropriate in any particular problem, and such points led to a lively discussion. The questions were not, of course, finally resolved, but the discussions gave all participants a rather clearer idea of the unsolved problems.

Another question about models which figured prominently in the discussion was the proper description for a nucleus in interaction with a fast neutron or proton. H. H. Barschall (Wisconsin) reported very extensive and interesting results about the scattering of neutrons in many nuclei, both as regards energy dependence and angular distribution, and this seems to agree remarkably well with a model proposed by Weisskopf in which the nucleus is treated as a sphere with a constant refractive index and absorption coefficient-a 'clouded crystal ball'-though the absorption coefficient used to fit the curves is surprisingly small.

Several different methods of estimating the radius of a nucleus were mentioned, the most extensive work being reported by $R$. Hofstadter (Stanford) on the scattering of $150-\mathrm{MeV}$. electrons by nuclei. These curves are interpreted directly as diffraction patterns. They would indicate an electric density distribution in the nucleus with a fairly strong maximum in the middle and a gradual decay rather than the usual picture of a uniform density with a sharply defined boundary. However, the distortion of the electron wave function by the electric field of the nucleus is large enough to throw doubt on the simple diffraction theory, and more work will have to be done to be sure of the interpretation of the experiments. The energy difference between the $2 p$ - and Is-levels of $\mu$-mesons near a nucleus also tends to indjcate that the generally accepted formula for the nuclear radius may not be reliable.

In considering most nuclear processes, it is proper to think of a nucleus as an isolated system, but one session of the conference centred around instances where the surrounding electrons, or even neighbouring atoms, are of major importance. Conversion of nuclear excitation energy through interaction with the atomic $K, L, M$... shells is a stock example; so also is the capture of an orbital electron by a nucleus. In addition to these, there were discussions of the directional correlations of successive radiations from a single atom. The anisotropy of the second radiation with respect to the direction of the first may be upset by a change of orientation of the nucleus while in the intermediate state. E. Heer (Zurich) described the effect of an external magnetic field on correlation between successive transitions in cadmium-111 (the product of the $\beta$-decay of indium111), from which the magnetic moment of the first excited state could be deduced. The anisotropy in 
the absence of an external field depends upon the crystalline state of the source; for a polycrystalline indium source, it shows a maximum value of 20 per cent at the melting point. Experiments with single (non-cubic) crystals showed a dependence of the observed anisotropy upon the orientation of the counters with respect to the crystal axes.

Other topics involving atomic processes were the elastic scattering of gamma-rays, in which scattering by bound electrons is coherent with nuclear scattering and the orientation of radioactive nuclei by magnetic fields at low temperatures, which has been achieved with elements that form Tutton salts.

In sessions devoted to experimental techniques, reports were given by D. Walker and J. H. Fremlin (Birmingham) of the processes by which multiplycharged ions (for example, ${ }^{14} \mathrm{~N}^{6+}$ ) can be accelerated in a cyclotron and of the types of nuclear reaction that such relatively heavy nuclei produce. Particular interest was shown in an impromptu description given by R. Birge (Berkeley) of the bubble-chamber experiments initiated by Glaser at Michigan, in which ionizing particles cause ebullition in a superheated liquid.

\section{SECOND INTERNATIONAL CONGRESS ON RHEOLOGY}

$\mathrm{T}$

HE Second International Congress on Rheology was held in Oxford during July 26-31 and was attended by some 245 rheologists accompanied by about fifty visitors. The proceedings commenced with an address on "Rheology for Mathematicians", given by the president of the Congress, Sir Geoffrey Taylor, who discussed various way in which mathematicians can help rheologists and the attitude of practical rheologists to the pure mathematician.

The technical programme of the Congress consisted of six invited lectures as well as a number of sectional meetings run in parallel. Nearly half of the fortynine contributed papers dealt with properties of high polymers, and these provided the subject-matter for Section $A$ for the whole of the Congress. A short (half-day) Section $C$ was concerned with problems of lubrication, all other contributions being grouped into a miscellaneous Section $B$.

In drawing up the programme, the aim of the organizing committee had been to cover the extended field of "the study of the deformation and flow of matter" as widely as possible. The subjects of the invited lectures illustrate this policy: sur l'effet éléctrovisqueux (Mme. A. Dobry-Duclaux, Paris); rheological problems in the fabrication of plastics (Dr. R. S. Spencer, Midland, Mich.); rheology and applied mechanics (Dr. R. N. J. Saal, Amsterdam); rheological behaviour and the molecular jumping mechanism (Prof. F. H. Müller, Marburg); water association and hydrogels (Mr. E. Forslind, Stockholm); and biological problems for the rheologist (Dr. P. Eggleton, Edinburgh). As well as many papers on plastics and high polymers generally, sectional papers covered a wide range of materials-..for example, clays, metals, gelatine gels, flour doughs, diamonds, greases, bitumens and protoplasm. Discussions ranged from methods of viscometry to the study of chronic bronchitis in man and the diagnosis of pregnancy in the cow. Papers were read, and discussions took place, in English, French and German.

Most of the papers were circulated as preprints before the meeting opened; all of them, together with summaries of the discussions which followed, will be published in book form by Butterworth's Publications, Ltd., London, and will appear, it is hoped, within about six months. In conjunction with the Congress there was a small exhibition of commercially available rheological apparatus, and an Oxford bookseller also arranged a special display of rheological books.

There were three evening meetings. At the first of these, a number of technical films was shown, including one illustrating Sir Geoffrey Taylor's own investigations on the dragging of anchors. Another evening was devoted to a discussion on international organization of rheology, at which the Intermational Union of Pure and Applied Physics was represented by its president, Prof. N. F. Mott (Bristol), the International Union of Chemistry by Mme. A. Dobry-Duclaux (Paris) and that of Theoretical and Applied Mechanics by Prof. J. M. Burgers (Delft). It was decided to form an International Committee on Rheology consisting initially of one representative each from all national groups devoted solely to the study of rheology, with power to co-opt individual rheologists from countries where no such groups as yet exist. The membership of the committee is temporary, until the national societies can elect their own representatives. Later in the Congress, the representatives of France (Prof. H. Weiss), Germany (Prof. F. H. Müller), Great Britain (Mr. A. G. Ward), Netherlands (Dr. R. N. J. Saal) and United States (Dr. R. S. Spencer) met under the chairmanship of Mr. Ward, and co-opted Prof. B. Gross (Brazil), Prof. M. Reiner (Israel) and Prof. A. Peterlin (Yugoslavia). It was resolved also to send invitations to rheologists in India, Japan and the Scandinavian countries. The purposes of the Committee were laid down as follows : "(1) To be a permanent body for organizing future International Congresses on Rheology, (2) to encourage new national organizations for the study of rheology, and (3) to act as co-ordinating body in other international co-operations in the field of rheology". Dr. R. N. J. Saal (Amsterdam) is acting as temporary secretary.

A third evening was devoted to a discussion on nomenclature and symbols. A number of reports formed a basis for the discussion, including that by Prof. J. M. Burgers and Dr. G. W. Scott Blair, published in 1949 under the auspices of the now defunct Joint Committee on Rheology. Dr. H. Leaderman (Washington, D.C.) reported on the still tentative but valuable "proposals for nomenclature for linear visco-elastic behavior" put forward by the Committee on Nomenclature of the (American) Society of Rheology. Following the discussion, certain recommendations were sent to Dr. Saal as secretary of the new International Committee.

At the Congress dinner, held in the Hall of Christ Church, Sir Ben Lockspeiser, as guest of honour, in reply to the toast of "The Guests", proposed by the president, summarized the history of the British Society of Rheology and recalled the First Congress held in Scheveningen in 1948. Dr. R. S. Spencer, president of the American Society, proposed "International Rheology", to which Mme. Dobry-Duclaux replied in French.

The Congress was closed by Prof. A. Peterlin (Yugoslavia), who expressed the gratitude of overseas rheologists to their British colleagues who had organized the Congress. Under the auspices of the newly formed International Committee, it is hoped that other equally pleasant and profitable congresses will be held in future years. G. W. ScotT BLarr 